\title{
An Efficient Finite Element Method for Treating Singularities in Laplace's Equation
}

\author{
Lorraine G. Olson, Georgios C. Georgiou, ${ }^{*}$ and William W. Schultz \\ Department of Mechanical Engineering and Applied Mechanics, \\ University of Michigan, Ann Arbor, Michigan 48109
}

Received June 16, 1989; revised February 2, 1990

\begin{abstract}
We present a new finite element method for solving partial differential equations with singularities caused by abrupt changes in boundary conditions or sudden changes in boundary shape. Terms from the local solution supplement the ordinary basis functions in the finite element solution. All singular contributions reduce to boundary integrals after a double application of the divergence theorem to the Galerkin integrals, and the essential boundary conditions are weakly enforced using Lagrange multipliers. The proposed method eliminates the need for high-order integration, improves the overall accuracy, and yields very accurate estimates for the singular coefficients. It also accelerates the convergence with regular mesh refinement and converges rapidly with the number of singular functions. Although here we solve the Laplace equation in two dimensions, the method is applicable to a more general class of problems. (C) 1991 Academic Press, Inc.
\end{abstract}

\section{INTRODUCTION}

Singularities often occur in models of engineering problems due to discontinuities in the boundary conditions or abrupt changes in the boundary shape. Two wellknown examples are the crack-tip problem in fracture mechanics [1] and the sudden-expansion problem in fluid mechanics [2].

When using numerical methods to solve problems with singularities, one must pay special attention to the singular regions. In both the finite difference and the finite element methods, local refinement is often employed near the singularity to achieve reasonable accuracy. However, the accuracy achieved and the rate of convergence are generally not uniform nor satisfactory $[1,3]$.

Incorporating the form of the singularity in a numerical scheme is generally more effective than mesh refinement. This idea has been successfully adopted in a variety of methods such as finite differences $[4,5]$, finite elements $[1,6-13]$, global elements $[14,15]$, and boundary elements $[16]$.

\footnotetext{
* Presently at Unité de Mécanique Appliquée, Université Catholique de Louvain, 2 Place du Levant, 1348 Louvain-la-Neuve, Belgium.
} 
The form of the singularity is obtained by a local analysis. The asymptotic $(r \rightarrow 0)$ solution for Laplace's equation in two dimensions is given by

$$
u(r, \theta)=\sum_{i=1}^{\infty} \alpha_{i} r^{\lambda_{i}} f_{i}(\theta),
$$

where $u$ is the dependent variable, $(r, \theta)$ are the polar coordinates centered at the singular point, $\alpha_{i}$ are the singular coefficients, $\lambda_{i}$ are the singularity exponents, and $f_{i}(\theta)$ represent the $\theta$ dependence of the eigensolution. The asymptotic solution satisfies the governing equation in the domain and the boundary conditions along the boundary segments adjacent to the singular point.

In this work, we focus on the finite element method, which is very popular in many engineering fields for solving problems with complex geometries. The local solution can be incorporated into a finite element scheme in two basic ways:

1. Singular finite element approach. Special elements are used in a small region around the singularity, while ordinary elements are used in the rest of the domain. The shape functions defined on the special elements take into account the known form of the singularity. This approach has one fundamental drawback: the size of the singular elements, and thus the region over which the singularity is given special attention, is reduced as the mesh is refined.

2. Singular basis function approach. A set of supplementary functions that reproduce the functional form of the leading terms of the singularity solution is added to the ordinary finite element expansion. In this approach, the singular representation is independent of the mesh refinement, and the singular coefficients are directly calculated.

Accurate estimates of the leading singular coefficients are often desirable, as in fracture mechanics [13] (the first coefficient is the stress intensity factor, a measure of the stress a body can endure before fracture occurs). For reviews on singular finite clement approaches, see Fix [13], Gallagher [17], and Gcorgiou [18].

In this paper we follow the singular basis function approach. The supplementary basis functions $W_{i}$ take the general form

$$
W_{i}=Q r^{\lambda_{i}} f_{i}(\theta)
$$

where $Q$ is an optional blending function. At least three kinds of blending functions appear in the literature:

1. Functions with two-zone blending $[1,7]$. For example,

$$
Q= \begin{cases}1, & 0 \leqslant r \leqslant R_{i} \\ P_{i}(r), & R_{i} \leqslant r \leqslant R_{0} \\ 0, & r \geqslant R_{0}\end{cases}
$$

where $R_{i}$ and $R_{0}$ may be chosen independently of the mesh size, and the polynomial 
$P_{i}$ smoothly forces the singular functions to zero at $r=R_{0}$. The functional form near the singular point is not affected by the blending, and no extra boundary terms appear in the finite element formulation.

2. Functions with one-zone blending $[6,19]$. Here, $Q$ modifies the singular terms even near the singular point. One choice is simply to let $R_{i}$ go to 0 in (3). Another choice is to conform the blending to the underlying mesh, e.g.,

$$
Q= \begin{cases}\left(1-x^{2}\right)\left(1-y^{2}\right), & x^{2} \leqslant H, y^{2} \leqslant H \\ 0, & \text { otherwise, }\end{cases}
$$

for a rectangular grid in cartesian coordinates with the singularity at the origin. Again, no extra boundary terms appear in the formulation if the boundaries are located at $x^{2}, y^{2} \geqslant H$, since the singular contributions are zero along the boundaries.

3. Exact function (no blending) [6]. In this case, $Q \equiv 1$. Additional boundary terms appear in the finite element formulation, since the singular functions are not zero along parts of the boundary. Also, the essential boundary conditions must be enforced separately.

Many researchers have used the singular basis function approach to solve a variety of problems such as the cracked-beam problem [13], the L-shaped membrane vibration problem [19], and reentrant-corner problems [20]. The two-zone and one-zone blending methods have two main advantages over other singular treatments: they are easy to program; and they are easily extended to nonlinear problems. However, the blending function introduces additional arbitrary parameters, contaminates the singular functions, and generally causes inaccurate estimates in the second and higher singular coefficients. In addition, a highaccuracy quadrature rule must be used to integrate the blended singular functions in the neighborhood of the singular point [1]. The unblended method avoids the singular function contamination problem, but requires separate enforcement of the essential boundary conditions and accurate quadrature rules to evaluate the integrals.

In this paper we present a new method with no blending function: the integrated singular basis function method (ISBFM). In the ISBFM, the analytical form of the asymptotic terms are used as the singular functions, and the volume integrals with singular contributions are reduced to boundary integrals by means of a double application of the divergence theorem. This method avoids the reduced accuracy associated with the blending function and eliminates the need to evaluate singular integrands.

We demonstrate the ISBFM on two Laplace-equation problems that have been studied extensively: the Motz problem [4] and the cracked-beam problem [19], described below.

The Motz problem. Figure 1 shows the geometry, governing equations, and boundary conditions for the Motz problem [4] as modified by Wait and Mitchell [7]; 


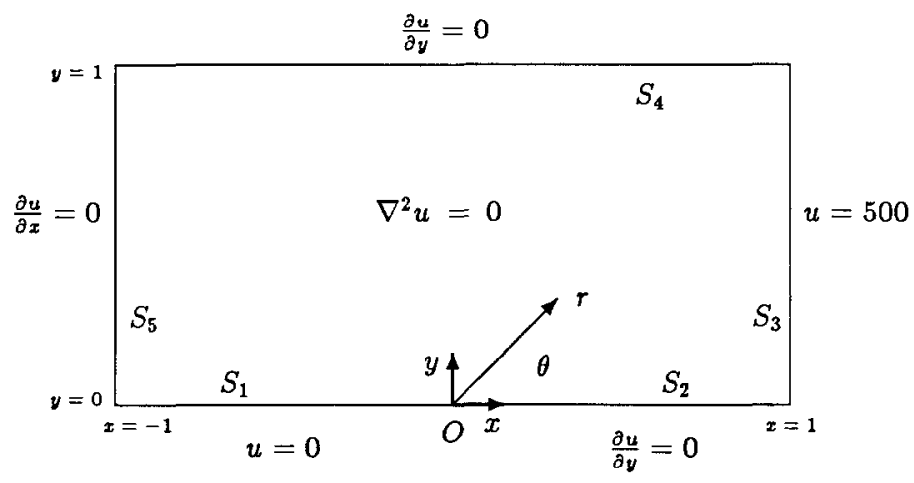

Fig. 1. The Motz problem.

this is considered a benchmark problem for testing the various singular approaches proposed in the literature. A singularity arises at $x=y=0$, where the boundary condition suddenly changes from $u=0$ to $\partial u / \partial y=0$. The local solution is given by [21]

$$
u(r, \theta)=\sum_{i=1}^{\infty} \alpha_{i} r^{(2 i-1) / 2} \cos \left[\left(\frac{2 i-1}{2}\right) \theta\right] .
$$

Whiteman [5] employed special finite difference methods to solve the problem, while Hendry and Delves [15] and Kermode et al. [14] used the global element method to determine the singular coefficients $\alpha_{i}$. Wait and Mitchell [7] used a combination of two-zone blended singular basis functions and mesh refinement and obtained disappointing results for the singular coefficients. The zones they employed were very small, and some of the singular functions were inappropriate for the Motz problem. Morley [6] applied both one-zone blending and no blending functions. While the singular treatments improved the solution, the actual values of the singular coefficients were not satisfactory.

Wigley [21] obtained very accurate estimates for the leading singular coefficients with an inherently iterative approach. He first generated an approximate numerical solution using finite differences and then estimated the first singular coefficient from this solution. Next, he modified the original problem by subtracting out the first singular term and again generated an approximate numerical solution to this modified problem, which he used to estimate the second singular coefficient, and so on. He obtained excellent results for the first several singular coefficients in the Motz problem as well as the cracked-beam problem.

The Cracked-Beam Problem. The second problem we examine is the crackedbeam problem $[13,21]$ illustrated in Fig. 2. (In the original problem, $\nabla^{2} v=-1$ and $v=0$ along $y=\frac{1}{2}$. The transformation $u=v+y^{2} / 2$ leads to the problem considered here.) Clearly, the form of the local solution is the same as that of the Motz problem. 
$u=0.125$

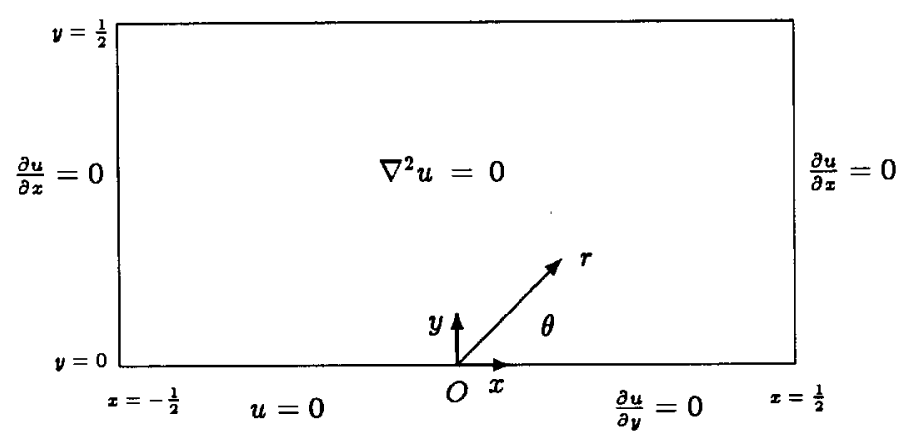

FIG. 2. The cracked-beam problem.

Fix et al. [19] used blended singular basis function approaches for this problem. They found that their method was efficient and moderately accurate. Fix [13] later reviewed singular basis function approaches and discussed the cracked-beam problem. As mentioned above, Wigley [21] obtained very accurate coefficients for the cracked-beam using an iterative method.

In the remainder of this paper, we discuss the solution of the two problems above with

1. Ordinary finite elements,

2. One- and two-zone blending functions (we refer to this method as the blended singular basis function method, BSBFM), and

3. The ISBFM.

In Section 2 we present the finite element formulation for all three methods. The results for the Motz problem, in Section 3, indicate that the accuracy of both the BSBFM and ISBFM is substantially better than that of the ordinary elements. With the BSBFM, we examine three different blendings and study the effects of the order of integration around the singular point and the size of the region over which the singular functions are defined. As mentioned above, high-order integration is not required with the ISBFM, and the singular functions are defined over the entire domain. The estimates of the leading coefficients with the ISBFM are more accurate than those with the BSBFM. The ISBFM yields an algebraic rate of convergence with mesh refinement, in agreement with the theoretical error estimates. It also converges rapidly with the number of singular functions. In Section 4 we solve the cracked-beam problem and confirm the accuracy and rate of convergence of the ISBFM. Finally, in Section 5 we summarize the conclusions. 


\section{Finite Element Formulation}

We now present the Galerkin finite element formulation for the Motz problem. The formulation for the cracked-beam problem is similar; thus, it is not included in this paper.

\subsection{Ordinary Finite Elements}

The unknown $u$ is expanded in terms of biquadratic basis functions $\Phi^{i}$ :

$$
u=\sum_{i=1}^{N_{u}} u_{i} \Phi^{i}
$$

where $N_{u}$ is the number of unknowns, and $u_{i}$ are the nodal values.

We apply Galerkin's method by weighting the governing equation with the basis functions $\Phi^{i}$ and then using the divergence theorem:

$$
\int_{S} \frac{\partial u}{\partial n} \Phi^{i} d S-\int_{V} \nabla u \cdot \nabla \Phi^{i} d V=0, \quad i=1,2, \ldots, N_{u}
$$

Here, $V$ is the physical domain, $S$ denotes its boundary (consisting of five different part as shown in Fig. 1), and $n$ is the outward normal from $S$. The boundary terms can be omitted because there are only essential and homogeneous natural boundary conditions, and (7) is simplified to

$$
-\int_{V} \nabla u \cdot \nabla \Phi^{i} d V=0, \quad i=1,2, \ldots, N_{u} .
$$

The equations for the essential boundary conditions along $S_{1}$ or $S_{3}$ replace the corresponding equations in (8). Equation (8) constitutes a symmetric and banded linear system of equations that is solved using standard subroutines.

\subsection{The Blended Singular Basis Function Method}

To the ordinary finite element expansion, we add the singular basis functions $W^{i}$,

$$
u=\sum_{i=1}^{N_{u}} u_{i} \Phi^{i}+\sum_{i=1}^{N_{\mathrm{SBF}}} \alpha_{i} W^{i}
$$

where $N_{\mathrm{SBF}}$ is the number of singular functions, and $\alpha_{i}$ are the unknown singular coefficients.

For comparison, we construct three different sets of blended singular functions:

$$
\text { BSBF1: } W^{i}= \begin{cases}r^{(2 i-1) / 2} \cos \frac{2 i-1}{2} \theta, & 0 \leqslant r \leqslant R / 2 \\ \frac{1}{R^{2}}(r-R)^{2}(a r-b) \cos \frac{2 i-1}{2} \theta, & R / 2 \leqslant r \leqslant R \\ 0, & r \geqslant R\end{cases}
$$


BSBF2: $W^{i}= \begin{cases}\frac{1}{R^{3}} r^{(2 i-1) / 2}(r-R)^{2}(2 r+R) \cos \frac{2 i-1}{2} \theta, & 0 \leqslant r \leqslant R \\ 0, & r \geqslant R\end{cases}$ BSBF3: $W^{i}= \begin{cases}r^{(2 i-1) / 2}\left(1-\frac{x^{2}}{H^{2}}\right)\left(1-\frac{y^{2}}{H^{2}}\right) \cos \frac{2 i-1}{2} \theta, & 0 \leqslant|x|,|y| \leqslant H \\ 0, & \text { elsewhere. }\end{cases}$

Here the radius $R$ and the length $H$ define the size of the singular domain. BSBF1 are functions with two-zone blending as suggested by Strang and Fix [1]. The coefficients $a$ and $b$ are determined by demanding continuity of $W^{i}$ and its first derivative at $r=R / 2$. BSBF2 and BSBF3 are functions with one-zone blending. As $r$ goes to zero, they all converge to the asymptotic solution.

Applying Galerkin's principle, we weight the governing equation by $\Phi^{i}$ and $W^{i}$. After using the divergence theorem we obtain

$$
\int_{S} \frac{\partial u}{\partial n} \Phi^{i} d S-\int_{V} \nabla u \cdot \nabla \Phi^{i} d V=0, \quad i=1,2, \ldots, N_{u},
$$

and

$$
\int_{S} \frac{\partial u}{\partial n} W^{i} d S-\int_{V} \nabla u \cdot \nabla W^{i} d V=0, \quad i=1,2, \ldots, N_{\mathrm{SBF}}
$$

Note that the total number of unknowns is now $N_{u}+N_{\mathrm{SBF}}$. The boundary terms in (10) and (11) can be omitted on those parts of the boundary where a homogeneous natural boundary condition is applied. The boundary terms on $S_{1}$ are ignored in (10) because we have essential conditions for $u_{i}$ and in (11) because $W^{i}$ are zero. Therefore,

$$
-\int_{V} \nabla u \cdot \nabla \Phi^{i} d V=0, \quad i=1,2, \ldots, N_{u},
$$

and

$$
-\int_{V} \nabla u \cdot \nabla W^{i} d V=0, \quad i=1,2, \ldots, N_{\mathrm{SBF}}
$$

Again, the equations for the essential boundary conditions along $S_{1}$ and $S_{3}$ replace the corresponding equations in (12). High-order integration is required for the terms involving singular functions in (13). Equations (12) and (13) constitute a symmetric linear system.

Using the singular functions destroys the banded structure of the stiffness matrix. The additional equations attach full rows and columns to the matrix that is 
otherwise banded about the main diagonal, resulting in an "arrow-shaped" matrix structure. Matrices of this structure are very often encountered when a basic boundary value problem is augmented with scalar constraints or is solved simultaneously with densely coupled algebraic equations [1,22]. Skyline solvers or extensions of other standard algorithms may be used in inverting the stiffness matrix to avoid extra operations during elimination as well as extra storage requirements. Strang and Fix [1] proposed a modified Choleski's factorization algorithm for arrow-shaped matrices, while Thomas and Brown [22] developed a modified LU-decomposition subroutine.

\subsection{The Integrated Singular Basis Function Method}

In the ISBFM we subtract the asymptotic terms directly from the governing equation. The singular functions are now identical to the form of the corresponding asymptotic expansion terms:

$$
W^{i}=r^{(2 i-1) / 2} \cos \left[\left(\frac{2 i-1}{2}\right) \theta\right] .
$$

Let $u^{s}$ be the singular part of $u$,

$$
u^{s}=\sum_{i-1}^{N_{\mathrm{SBF}}} \alpha_{i} W^{i},
$$

and $u^{*}$ be the part of the solution approximated by the ordinary finite element expansion,

$$
u^{*}=u-u^{s}
$$

Note that $u^{s}$ satisfies the governing equation and the boundary conditions along $y=0$, and the original problem is transformed to that shown in Fig. 3 .

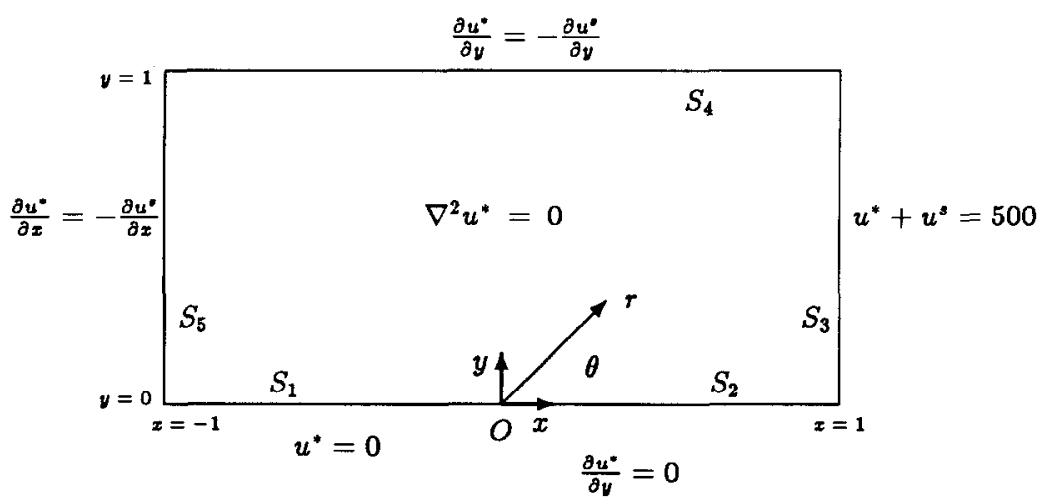

FIG. 3. The modified Motz problem. 
Again we use the Galerkin method (and the divergence theorem) to obtain

$$
\int_{S} \frac{\partial u^{*}}{\partial n} \Phi^{i} d S-\int_{V} \nabla u^{*} \cdot \nabla \Phi^{i} d V=0, \quad i=1,2, \ldots, N_{u},
$$

and

$$
\int_{S} \frac{\partial u^{*}}{\partial n} W^{i} d S-\int_{V} \nabla u^{*} \cdot \nabla W^{i} d V=0, \quad i=1,2, \ldots, N_{\mathrm{SBF}}
$$

To reduce the singular volume integrals of $(18)$ to boundary integrals, we apply the divergence theorem once morc:

$$
\int_{V} \nabla u^{*} \cdot \nabla W^{i} d V=\int_{S} u^{*} \frac{\partial W^{i}}{\partial n} d S-\int_{V} u^{*} \nabla^{2} W^{i} d V .
$$

The volume integral on the right-hand side of (19) is zero, since the $W^{i}$ satisfy Laplace's equation, and (18) becomes

$$
\int_{S}\left(\frac{\partial u^{*}}{\partial n} W^{i}-u^{*} \frac{\partial W^{i}}{\partial n}\right) d S=0, \quad i=1,2, \ldots, N_{\mathrm{SBF}} .
$$

The boundary terms are not ignored, since $\partial u^{*} / \partial n=-\partial u^{s} / \partial n$. To impose the originally essential boundary condition on $S_{3}$, we employ Lagrange multipliers $\lambda_{u}^{i}$ expanded in terms of quadratic basis functions $M^{i}$ :

$$
\lambda_{u}=\frac{\partial u^{*}}{\partial x}=\sum_{i=1}^{N_{y}} \lambda_{u}^{i} M^{i}
$$

$N_{y}$ is the number of nodes on $S_{3}$. (Bertsekas [23] and Babuska [24] discuss the general use of Lagrange multipliers with constrained optimization problems and finite elements.) The final equations are

$$
\begin{gathered}
\int_{S_{3}} \lambda_{u} \Phi^{i} d y-\int_{S_{4}} \frac{\partial u^{s}}{\partial y} \Phi^{i} d x-\int_{S_{5}} \frac{\partial u^{s}}{\partial x} \Phi^{i} d y-\int_{V} \nabla u^{*} \cdot \nabla \Phi^{i} d V=0, \\
\int_{S_{3}}\left(\lambda_{u} W^{i}+u^{s} \frac{\partial W^{i}}{\partial x}\right) d y-\int_{S_{4}}\left(\frac{\partial u^{s}}{\partial y} W^{i}+u^{*} \frac{\partial W^{i}}{\partial y}\right) d x-\int_{S_{5}}\left(\frac{\partial u^{s}}{\partial x} W^{i}+u^{*} \frac{\partial W^{i}}{\partial x}\right) d y \\
=500 \int_{S_{3}} \frac{\partial W^{i}}{\partial x} d y, \quad i=1,2, \ldots, N_{\mathrm{SBF}}
\end{gathered}
$$

and

$$
\int_{S_{3}}\left(u^{*}+u^{s}\right) M^{i} d y=500 \int_{S_{3}} M^{i} d y, \quad i=1,2, \ldots, N_{y} .
$$


The total number of unknowns is now $N_{u}+N_{\mathrm{SBF}}+N_{y}$. The linear system of equations defined by (22)-(24) is symmetric. Since the singular functions satisfy the boundary conditions near the singular point, all of the surface integrands are nonsingular and are evaluated with an ordinary Gauss-Legendre quadrature. As with the BSBFM, the banded structure of the stiffness matrix is destroyed in the ISBFM. This, along with the increase in the number of unknowns, results in some additional computational costs for a given underlying mesh. However, since the accuracy is high with the ISBFM, mesh refinement may not be necessary.

Neither of the singular function techniques (BSBFM or ISBFM) require a rectangular domain.

Convergence estimates. For convergence studies on the ISBFM, we calculate the variational indicator $\Pi$. For Laplace's equation, $\Pi$ may be written as [25]

$$
\Pi=\frac{1}{2} \int_{V} \nabla u \cdot \nabla u d V-\int_{S} u \frac{\partial u}{\partial n} d S .
$$

The error measure $\left(\Pi_{h}-\Pi_{\mathrm{ex}}\right)^{1 / 2}$ is of the same order as the energy error and satisfies the inequality

$$
\left(\Pi_{h}-\Pi_{\mathrm{ex}}\right)^{1 / 2} \leqslant\left\|u_{\mathrm{ex}}-u_{h}\right\|_{1}
$$

The subscript $h$ denotes quantities calculated with a mesh size $h$, the subscript ex indicates the exact solution, and the norm $\|u\|_{m}$ has the standard definition [26]. It is well known that the energy error satisfies

$$
\left\|u_{\mathrm{ex}}-u_{h}\right\|_{1} \leqslant C h^{\mu}\left\|u_{\mathrm{ex}}\right\|_{m}
$$

with

$$
\mu=\min \{k, m-1\},
$$

where $C$ is a constant, $\mu$ is the convergence rate, $k$ is the order of the finite element polynomial approximation (2, in this work), and $m$ is the order of the generalized Sobolev space of the exact solution [3]. The order $m$ is a measure of the regularity of the solution, or an inverse measure of the singularity strength, given by

$$
m=1+\lambda_{1},
$$

where $\lambda_{1}$ is the exponent in the first term of the local solution (1). For example, $m=\frac{3}{2}$ for the Motz problem and the cracked-beam problem and, according to (28), if no singular functions are used the convergence rate is $\mu=0.5$, independent of the order of the finite element approximation $k$. Subtracting the first term of the local solution changes $m$ to $\frac{5}{2}$, and the rate of convergence will be $\mu=1.5$. Subtracting two or more singular functions results in quadratic convergence (for $k=2$ ). 


\section{Results for the Motz Problem}

In order to compare the three approaches for the Motz problem (i.e., ordinary finite elements, blended singular basis functions, and integrated singular basis functions), we first establish the best integration order and singular domain for the BSBFM. This question is addressed in Section 3.1. Section 3.2 examines the convergence of the ISBFM with mesh refinement and $N_{\mathrm{SBF}}$. Section 3.3 compares the actual solutions for ordinary finite elements, BSBFM, and ISBFM, and examines the singular coefficients for BSBFM and ISBFM.

\subsection{A Comparison of Blended Singular Basis Function Methods}

Unlike the ISBFM, the BSBFM requires high-order integration if converged results are to be obtained. To study the effect of the order of the Gauss-Legendre quadraturc, we first use a uniform mesh with $16 \times 8$ elements, set $R$ and $H$ (the size of the singular domain) equal to the mesh size, and use $N_{\mathrm{BSF}}=1$. The computed values of $\alpha_{1}$ are plotted in Fig. 4. BSBF1 and BSBF2 give poor estimates for $\alpha_{1}$, whereas BSBF3 appears to converge to the exact value as the order of integration increases. We have found satisfactory results when high-order integration is employed only over the two elements sharing the singular point. For all the BSBFM results hereafter, each element adjacent to the singular point is divided into 64 rectangles over which a $15 \times 15$ Gauss quadrature is used.

The effect of mesh refinement on the first coefficient $\alpha_{1}$ is shown in Fig. 5; here

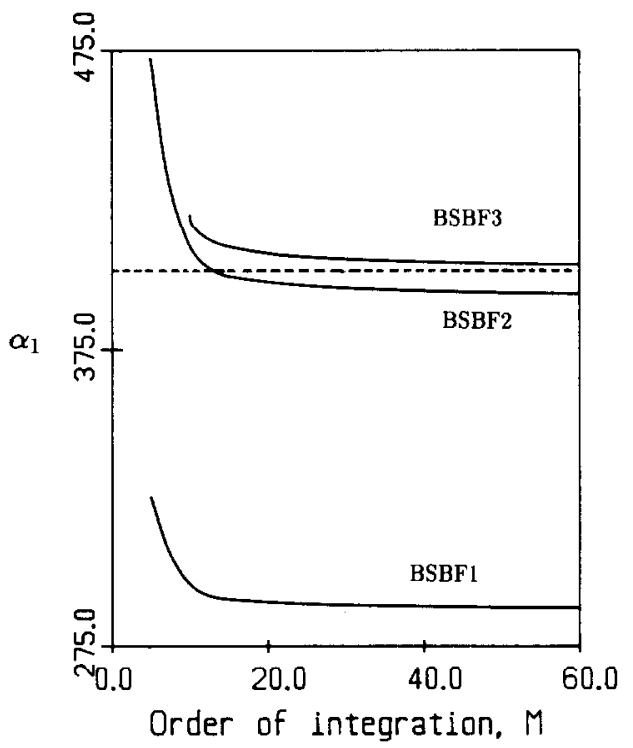

FIG. 4. The coefficient $\alpha_{1}$ as a function of the order of integration $m$ (16 $\times 8$ mesh; $R$ and $H$ equal to meshsize; ----: analytical value). 


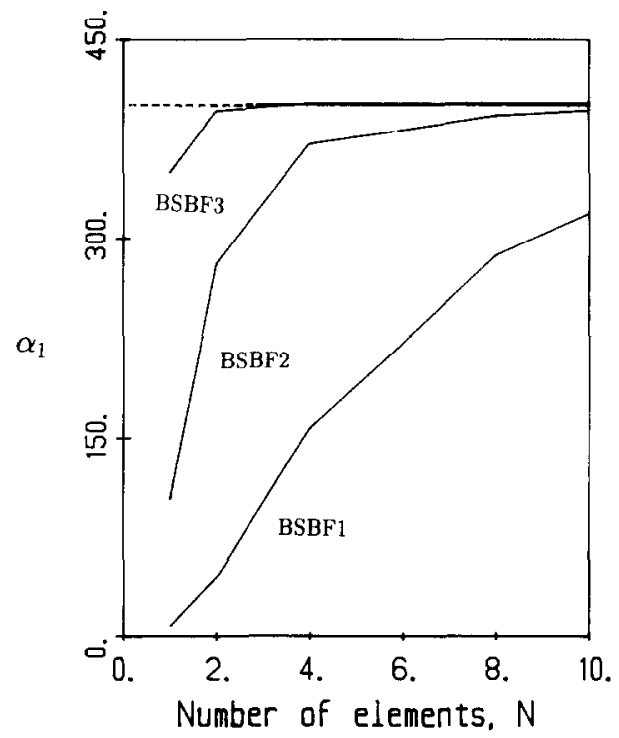

FIG. 5. Effect of mesh refinement on $\alpha_{1}$ ( $R$ or $H$ are equal to the meshsize; - - - analytical value).

we use different uniform meshes and keep $R$ (or $H$ ) equal to the mesh size. Each mesh consists of $2 N \times N$ square elements, where $N$ is the number of elements in the $y$ direction. The results appear to approach the analytical value with mesh refinement in all cases. Among the blended singular functions used, BSBF3 again appears to give the best results.

The singular domain in the foregoing analysis includes only the two elements sharing the singular point, or parts of them. It seems that larger values of $R$ (or $H$ ) would allow the ordinary finite element expansion coefficients to adjust more effectively to the addition of one or more singular terms. To investigate this, we use a $16 \times 8$ element mesh, varying $R$ from $\frac{1}{8}$ up to 1 . The computed coefficients are plotted in Fig. 6. We observe that the results with BSBF2 and BSBF3 converge very close to the exact value. The results with BSBF1 exhibit analogous behavior but higher values of $R$ (or more elements within the singular domain) are required to reach a plateau.

We conclude that BSBF3 with $H$ equal to the size of the domain is the best of the blended singular basis functions examined. Although these tests were conducted on the Motz problem, it seems reasonable to suppose that BSBF3 would also prove superior in other applications. All the results with the BSBFM hereafter are obtained with the BSBF3.

\subsection{ISBFM Convergence}

Figure 7 shows the convergence rate achieved for the ISBFM: $\mu=0.50$ when no singular functions are used, $\mu=1.54$ when $N_{\mathrm{BSF}}=1$, and $\mu=1.9$ when $N_{\mathrm{SBF}}=2$. 


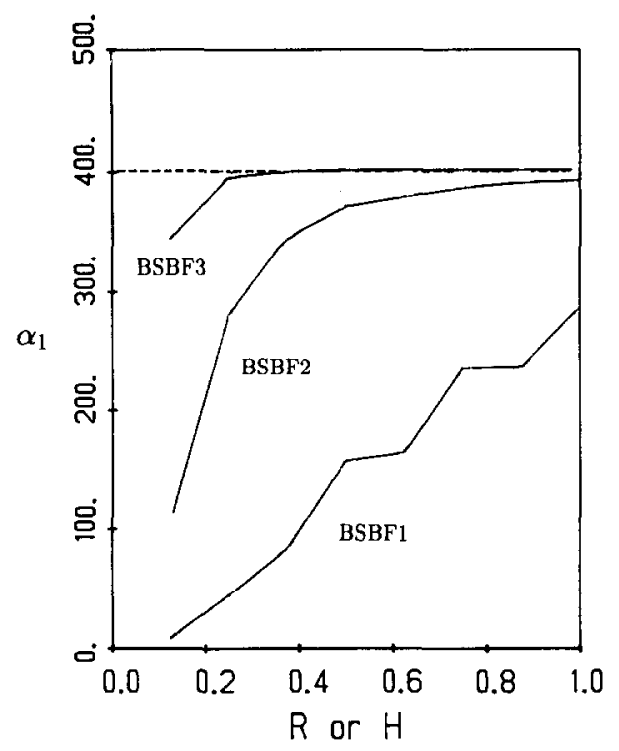

FIG. 6. Effect of the size of the singular domain ( $R$ or $H$ ) on the first coefficient $\alpha_{1}(16 \times 8$ uniform mesh; ----: analytical value).

(Recall that Eq. (28) indicates that the rate of convergence of $\left(\Pi_{h}-\Pi_{\mathrm{ex}}\right)^{1 / 2}$ should be $\mu=0.5$ when no singular functions are used; when one singular function is used, $\mu$ should increase to 1.5; and with two or more singular functions, $\mu=2.0$.) For the problems considered in this paper, no analytical expression for $\Pi$ is available; therefore, we use the estimate from a very fine mesh $(32 \times 16$ elements, 20 singular

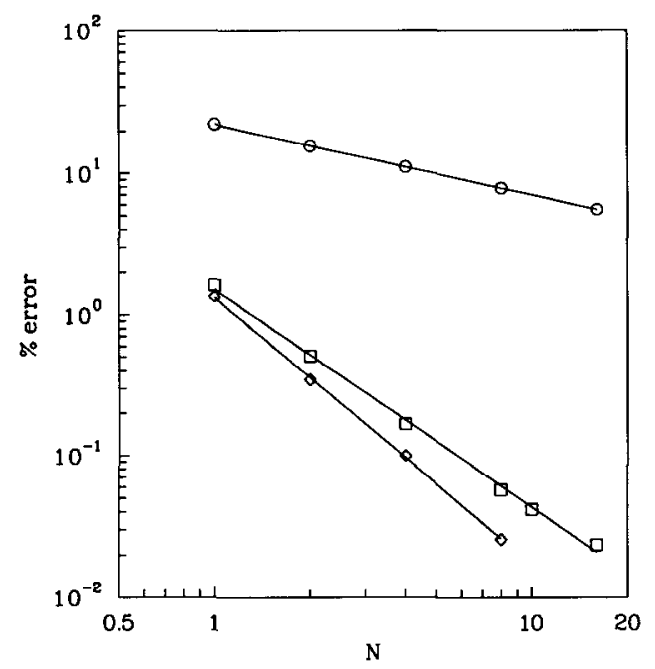

FIG. 7. Convergence with mesh refinement for the Motz problem ( $O$ : no singular functions; $\square$ : one singular function, $\diamond:$ two singular functions). 


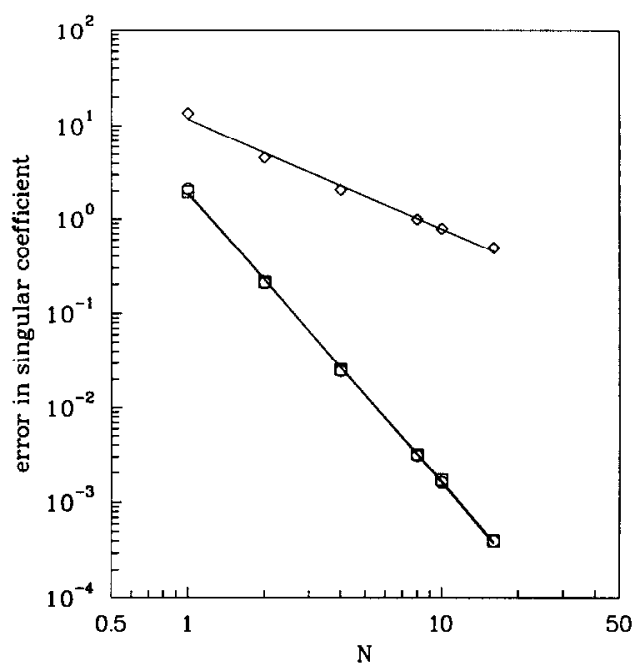

Fig. 8 . Convergence of singular coefficients with mesh refinement for the Motz problem $\left(0: \alpha_{1}\right.$ with one singular function, $\square: \alpha_{1}$ with two singular functions, $\diamond: \alpha_{2}$ with two singular functions).

functions) as the "exact" value of $\Pi$. The slight discrepancies from the theoretical convergence rates may be partially due to this approximation for $\Pi$. As indicated by (28), higher convergence rates can be achieved using higher-order elements.

Figure 8 shows the rate of convergence of the singular coefficients as the mesh is refined. With one singular basis function, $\alpha_{1}$ converges essentially cubically. When two singular basis functions are used, the rate of convergence of $\alpha_{2}$ is approximately linear, while the rate of convergence of $\alpha_{1}$ is essentially unchanged.

Figure 9 shows the rapid (roughly exponential) convergence of $\left(\Pi_{h}-\Pi_{\mathrm{ex}}\right)^{1 / 2}$

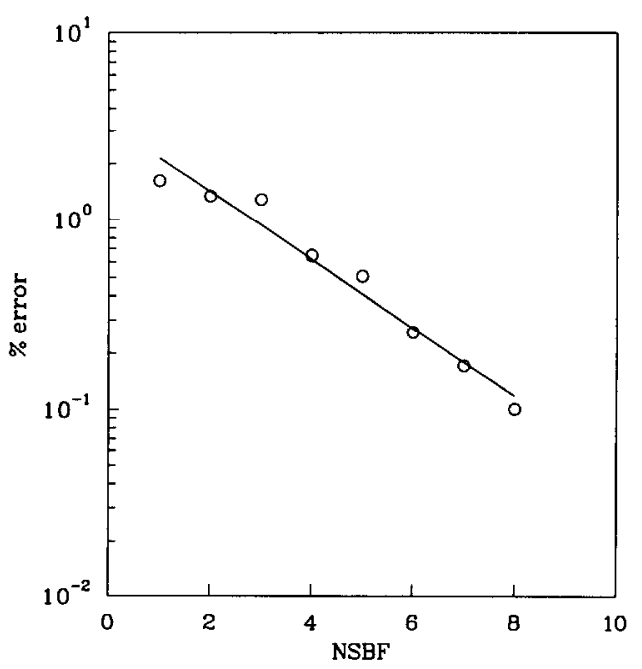

FIG. 9. Convergence with the number of singular functions for the Motz problem $(2 \times 1$ mesh). 
TABLE I

Leading Coefficients for the Motz Problem $\left(N_{\mathrm{SBF}}=20\right.$, $16 \times 8 \mathrm{Mesh})$

\begin{tabular}{rrrc}
\hline$i$ & ISBFM & Wigley [21] & Exact [27] \\
\hline 1 & 401.1625 & 401.163 & 401.1625 \\
2 & 87.6559 & 87.655 & 87.65592 \\
3 & 17.2379 & 17.238 & 17.23792 \\
4 & -8.0712 & -8.071 & -8.0712 \\
5 & 1.4403 & 1.440 & \\
6 & 0.3310 & 0.331 & \\
7 & 0.2754 & 0.275 & \\
8 & -0.0869 & -0.087 & \\
9 & 0.0336 & 0.0336 & \\
10 & 0.0154 & 0.0154 & \\
11 & 0.0073 & 0.0073 & \\
12 & -0.0032 & -0.0032 & \\
13 & 0.0012 & 0.0012 & \\
14 & 0.0005 & 0.0005 & \\
\hline
\end{tabular}

versus the number of singular terms for the ISBFM. The convergence with the number of singular functions is considerably faster than the convergence with mesh refinement.

In Table I we list the singular coefficients calculated with the ISBFM using 20 singular functions and a $16 \times 8$ mesh. They are in close agreement with the exact values available [27] and with Wigley's results for the higher coefficients [21].

At some point, we expect the condition number of the stiffness matrices will become too large to permit an accurate solution of the equations. Table II shows the condition number estimates, $\kappa$, for a $2 \times 1$ mesh wih various numbers of singular functions. With 35 coefficients, the condition number is quite poor and the

TABLE II

Condition Numbers for the Motz Problem (ISBFM, $2 \times 1$ mesh)

\begin{tabular}{rc}
\hline$N_{\mathrm{SBF}}$ & $1 / \kappa$ \\
\hline 5 & $0.40 \times 10^{-5}$ \\
10 & $0.60 \times 10^{-7}$ \\
15 & $0.11 \times 10^{-8}$ \\
20 & $0.47 \times 10^{-10}$ \\
25 & $0.21 \times 10^{-11}$ \\
30 & $0.44 \times 10^{-13}$ \\
35 & $0.12 \times 10^{-14}$ \\
40 & $0.48 \times 10^{-16}$ \\
\hline
\end{tabular}


numerical results begin to diverge. It is interesting to note that when we use 30 singular functions, the ordinary contribution to the solution is zero to four decimal places.

\subsection{Comparison between Ordinary Elements, BSBFM, and ISBFM}

Initially, we study the effect of increasing the number of singular functions for both the BSBFM and the ISBFM with a $16 \times 8$-element mesh. As shown in Tables III and IV, increasing the number of functions for the ISBFM improves the accuracy of the leading coefficients, which appear to converge to the analytical values with increasing $N_{\mathrm{SBF}}$. With the BSBFM, the first coefficient remains essentially constant as we increase the number of singular functions, and the estimates of the higher coefficients are poor.

The disappointing results with the BSBFM for the higher coefficients may be due to contamination from the blending; extra higher-order terms that do not satisfy the governing equation are introduced with every $W^{i}$. For example, with the BSBF3,

$$
\begin{aligned}
W^{1} & =r^{1 / 2}\left(1-x^{2}\right)\left(1-y^{2}\right) \cos \frac{\theta}{2} \\
& =r^{1 / 2} \cos \frac{\theta}{2}-r^{5 / 2} \cos \frac{\theta}{2}+r^{7 / 2} \cos \frac{\theta}{2} \sin ^{2} \theta \cos ^{2} \theta .
\end{aligned}
$$

Only the $r^{1 / 2} \cos (\theta / 2)$ term should be associated with $\alpha_{1}$, although $\alpha_{1}$ multiplies all three terms in BSBF3. Therefore, one can expect a good estimate only for the first expansion coefficient, as noted by Whiteman [20], Morley [6], and Wait and Mitchell [7].

Table $\mathrm{V}$ compares the values of the solution $u$ at various points of the domain for the ordinary finite element method (OFE), the BSBFM, and the ISBFM. We observe that both the BSBFM and the ISBFM yield improved results compared to the ordinary elements. The ISBFM predictions, however, are closer to the exact values.

TABLE III

Values of the Leading Singular Coefficients for the Motz Problem from the BSBFM $(16 \times 8$ Uniform Mesh)

\begin{tabular}{cccccc}
\hline$N_{\text {BSF }}$ & $\alpha_{1}$ & $\alpha_{2}$ & $\alpha_{3}$ & $\alpha_{4}$ & $\alpha_{5}$ \\
\hline 1 & 402.21518 & & & & \\
2 & 402.21517 & 10.867980 & & & \\
3 & 402.21516 & 15.206817 & -3.563013 & & \\
4 & 402.21590 & 15.206704 & -3.562949 & -5.609400 & \\
5 & 402.21758 & 15.206599 & -3.562890 & -6.309102 & -2.677194 \\
Exact & 401.1625 & 87.65592 & 17.23792 & -8.0712 & \\
\hline
\end{tabular}


TABLE IV

Values of the Leading Singular Coefficients for the Motz Problem from the ISBFM

(16×8 Uniform Mesh)

\begin{tabular}{cccccc}
\hline$N_{\mathrm{SBF}}$ & $\alpha_{1}$ & $\alpha_{2}$ & $\alpha_{3}$ & $\alpha_{4}$ & $\alpha_{5}$ \\
\hline 1 & 401.15943 & & & & \\
2 & 401.15932 & 86.662605 & & & \\
3 & 401.15932 & 87.620499 & 14.603352 & & \\
4 & 401.16197 & 87.620963 & 14.601024 & -7.475231 & \\
5 & 401.16224 & 87.620202 & 14.604841 & -7.470403 & 1.224633 \\
Exact & 401.1625 & 87.65592 & 17.23792 & -8.0712 & \\
\hline
\end{tabular}

TABLE V

Solution of the Motz Problem at Various Points Compared with Values from the Literature $\left(16 \times 8 \mathrm{Mesh}, N_{\mathrm{SBF}}=1\right)$

\begin{tabular}{crrccc}
\hline$\left(x_{i}, y_{i}\right)$ & OFE & BSBFM & ISBFM & Exact [27] & Wigley [21] \\
\hline$(-6 / 7,6 / 7)$ & 90.964 & 91.342 & 91.341 & 91.34 & 91.343 \\
$(-2 / 7,2 / 7)$ & 78.053 & 78.560 & 78.559 & 78.56 & 78.559 \\
$(0,2 / 7)$ & 140.477 & 141.562 & 141.560 & 141.6 & 141.560 \\
$(2 / 7,2 / 7)$ & 242.783 & 243.814 & 243.812 & 243.8 & 243.812 \\
$(0,1 / 7)$ & 102.056 & 103.772 & 103.768 & 103.77 & 103.768 \\
$(-1 / 28,1 / 28)$ & 31.770 & 33.594 & 33.590 & 33.59 & 33.592 \\
$(0,1 / 28)$ & 50.261 & 53.197 & 53.190 & 53.19 & 53.186 \\
$(1 / 28,1 / 28)$ & 79.286 & 83.682 & 83.672 & 83.67 & 83.671 \\
$(1 / 28,0)$ & 72.264 & 76.412 & 76.403 & 76.41 & 76.408 \\
$(3 / 28,0)$ & 131.740 & 134.452 & 134.447 & & 134.447 \\
$(1 / 7,0)$ & 154.096 & 156.487 & 156.483 & 156.48 & 156.483 \\
\hline
\end{tabular}

TABLE VI

Leading Coefficients for the CrackedBeam Problem $\left(N_{\mathrm{SBF}}=20,16 \times 8 \mathrm{Mesh}\right)$

\begin{tabular}{rrr}
\hline$i$ & ISBFM & Wigley [21] \\
\hline 1 & 0.191119 & 0.19112 \\
2 & -0.118116 & -0.11811 \\
3 & -0.000000 & 0.00000 \\
4 & -0.000000 & 0.00000 \\
5 & -0.012547 & -0.01256 \\
6 & -0.019033 & -0.01905 \\
7 & 0.000000 & \\
\hline
\end{tabular}


TABLE VII

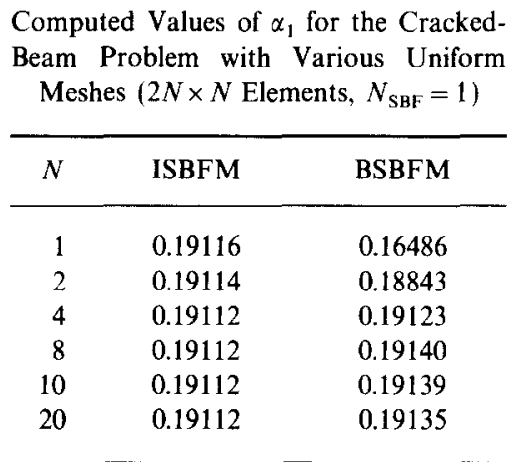

\section{Results for the Cracked-Beam Prublem}

As for the Motz problem, the ISBFM accelerates the convergence in agreement with (28) and gives very accurate estimates for the higher coefficients. The computed rate of convergence of $\left(\Pi_{h}-\Pi_{\mathrm{ex}}\right)^{1 / 2}$ with mesh refinement for the crackedbeam problem ( $\mu=0.5$ when no singular functions are used; $\mu=1.6$ when $N_{\mathrm{SBF}}=1$; and $\mu=2.0$ when $N_{\mathrm{SBF}}=2$ ) is similar to that obtained for the Motz problem. Table VI shows the first seven singular coefficients, which agree well with Wigley's iterative results [21]. Table VII compares the calculated values of $\alpha_{1}$ from the ISBFM and the BSBFM as the underlying mesh is refined. The ISBFM again converges significantly faster than the BSBFM. Finally, the values of $u$ obtained with the ordinary finite element method, the BSBFM, and the ISBFM are compared with results from the literature in Table VIII. As in the Motz problem, both the BSBFM and the ISBFM yield improved results compared to the ordinary element method.

TABLE VIII

Solution of the Cracked-Beam Problem at Various Points Compared with Results from the Literature $\left(16 \times 8 \mathrm{Mesh}, N_{\mathrm{SBF}}=1\right)$

\begin{tabular}{cccccc}
\hline$\left(x_{i}, y_{i}\right)$ & OFE & BSBFM & ISBFM & Fix et al. [19] & Wigley [21] \\
\hline$(0,1 / 24)$ & 0.026192 & 0.027431 & 0.027429 & 0.027425 & 0.027428 \\
$(-11 / 24,1 / 4)$ & 0.032847 & 0.032878 & 0.032879 & 0.032877 & 0.032878 \\
$(11 / 24,1 / 4)$ & 0.070657 & 0.070844 & 0.070844 & 0.070844 & 0.070844 \\
\hline
\end{tabular}




\section{Concluding Remarks}

The proposed integrated singular basis function approach (ISBFM) eliminates the need for high-order integration, improves the overall accuracy, and yields very accurate estimates for the singular coefficients. It also accelerates the convergence with mesh refinement, in agreement with theory; the same rate of convergence as for regular problems is achieved by including a sufficient number of singular functions. For a fixed mesh, convergence with the number of singular functions is very rapid. Although we have demonstrated the method only on examples involving singularities in the 2D Laplace's equation in rectangular domains, the approach is quite general and can be applied to arbitrary geometries and a wide range of governing equations. The extension of the method to fluid flow problems in complex geometries is currently under investigation.

\section{ACKNOWLEDGMENTS}

This work was partially supported by The Ocean Engineering Division of the Office of Naval Research, Contract N00014-87-0509, and the National Science Foundation, Contract DMC-8716766.

\section{REFERENCES}

1. G. Strang and G. J. Fix, An Analysis of the Finite Element Method (Prentice-Hall, Englewood Cliffs, NJ, 1973).

2. G. C. Georgiou, W. W. Schultz and L. G. Olson, Int. J. Numer. Methods Fluids 10, 357 (1990).

3. G. F. Carey and J. T. Oden, Finite Elements. A Second Course, Vol. II (Prentice-Hall, Englewood Cliffs, NJ, 1983).

4. H. Motz, Q. Appl. Math. 4, 371 (1946).

5. J. R. Whiteman, Proc. R. Soc. London A 323, 271 (1971).

6. L. S. D. MoRley, Phil. Trans. R. Soc. London A 275, 463 (1973).

7. R. Wait AND A. R. Mirchell, J. Comput. Phys. 8, 45 (1971).

8. R. WAIT, Comput. Methods Appl. Mech. Eng. 13, 141 (1978).

9. D. M. TraceY, Eng. Fracture Mech. 3, 255 (1971).

10. G. C. Georgiou, L. G. Olson, W. W. Schultz, and S. SaGan, Int. J. Numer. Methods Fluids 9, 1353 (1989).

11. G. C. Georgiou, L. G. Olson, AND W. W. Schultz, in Finite Element Analysis in Fluids, edited by T. J. Chung and G. R. Karr (UAH Press, Huntsville, AL, 1989).

12. G. Fix, J. Math. Mech. 18, No. 7, 645 (1969).

13. G. J. Fix, Finite Elements. Theory and Application, Chap. 3, edited by D. L. Dwoyer, M. Y. Hussaini, and R. G. Voigt (Springer-Verlag, New York, 1988).

14. M. Kermode, A. McKerrell, and L. M. Delves, Comput. Methods Appl. Mech. Eng. 50, 205 (1985).

15. J. A. Hendry and L. M. Delves, J. Comput. Phys. 33, 33 (1979).

16. D. B. Ingham and M. A. Kelmanson, Boundary Integral Equation Analyses of Singular, Potential and Biharmonic Problems (Springer-Verlag, Berlin, 1984).

17. H. Gallagher, in Proceedings of Symposium on Finite Element Method, Beijing, China (Science Press, Beijing, 1982).

18. G. Georgiou, Ph.D. Thesis, University of Michigan, Ann Arbor, 1989. 
19. G. J. Fix, S. Gulati, and G. I. Wakofr, J. Comput. Phys. 13, 209 (1973).

20. J. R. Whiteman, Finite Elements in Fluids, Vol. 2, Chap.6, edited by R. H. Gallagher, J. T. Oden, C. Taylor, and O. C. Zienkiewicz (Wiley, New York, 1985).

21. N. M. Wigley, J. Comput. Phys. 78, 369 (1988).

22. P. D. Thomas and R. A. Brown, Int. J. Numer. Methods Eng. 24, 1451 (1987).

23. D. P. BertsekAS, Constrained Optimization and Lagrange Multiplier Methods (Academic Press, New York, 1982)

24. I. BABUSKA, Numer. Math. 20, 179 (1973).

25. K. J. Bathe, Finite Element Procedures in Engineering Analysis (Prentice-Hall, Englewood Cliffs, NJ, 1982).

26. F. Brezzi and G. Gilardi, Finite Element Handbook, Chap. 2 edited by H. Kardestuncer (McGraw-Hill, New York, 1987).

27. J. B. Rosser and N. Papamichaed, MRC Technical Summary, Rep. 1405, University of Wisconsin, 1975. 